\title{
PANCREATIC METAPLASIA OF THE GASTRIC MUCOSA IN CHILD
}

\author{
Raluca Damian', Cristina Oana Marginean ${ }^{2,3}$, Nicoleta Suciü ${ }^{3}$, \\ Maria Oana Marginean ${ }^{3}$, Simona Mocan ${ }^{4}$, Lorena Elena Melit ${ }^{2,3}$ \\ ${ }^{1}$ Neonatology Clinic 1, Clinical Emergency Hospital, Targu-Mures \\ ${ }^{2}$ Pediatrics Clinic 1, Clinical Emergency Hospital, Targu-Mures \\ ${ }^{3}$ University of Medicine and Pharmacy, Targu-Mures \\ ${ }^{4}$ Department of Pathology, Clinical Emergency Hospital, Targu-Mures
}

\begin{abstract}
Pancreatic metaplasia is defined as the development of pancreatic tissue in an abnormal location and without direct connection to the pancreas. The heterotopic pancreatic tissue is most frequently noticed in the antral gastric mucosa, duodenum and jejunum. We present the case of a 13-year-old patient, admitted in Pediatrics Clinic 1 Targu-Mures, for recurrent abdominal pain and heartburns. The laboratory tests pointed out lymphocytosis $(41 \%)$ with monocytosis $(8.9 \%)$, eosinophilia $(4.9 \%)$, an increased number of reticulocytes $(20 \%)$, a decreased serum level of triglycerides and a value above the superior limit for calcium and magnesium. The abdominal ultrasound did not reveal any pathological modifications. The superior digestive endoscopy showed a granular gastric mucosa, therefore we took a biopsy of the antral mucosa, The pathological exam pointed out a fragment of pancreatic metaplasia in the antral gastric mucosa. The evolution was favorable with diet and treatment for the associated lesions. The particularity of the case consists in the presence of pancreatic metaplasia of the gastric mucosa in a patient with recurrent abdominal pain and heartburns, without significant familial or personal history.
\end{abstract}

Keywords: pancreatic metaplasia, child, abdominal pain, heartburn

\section{INTRODUCTION}

Pancreatic metaplasia of the gastric mucosa represents the process in which the normal mucosa of the stomach is replaced with pancreatic structures that mimic the structure of the pancreatic acinus $(1,2)$. Pancreatic metaplasia of the gastric mucosa was described for the first time in 1993, by Doglioni. Its incidence, in the last years, has increased (3). Until now, the causes that lead to pancreatic metaplasia in the gastric mucosa couldn't be described exactly. If in adult, pancreatic metaplasia is frequently associated with a chronic inflammatory processes, such as gastritis or ulcer, in children, the presence of pancreatic metaplasia seems to be caused by a disorder of gastric mucosa development and differentiation. Another factor that is supposed to lead to this type of metaplasia in the gastric mucosa is the infection and injury produced by the bacteria Helicobacter Pylori (4). Histologically, pancreatic metaplasia of the gastric mucosa ap- pears as areas of metaplasia, localized in the inferior third of the gastric chorion and it contains pancreatic acinar cells, disposed as nests or lobules, with variable sizes of up to $1.7 \mathrm{~mm}$ diameter. The metaplasia tissue can merge with the gastric fundic glands. The acinar pancreatic cells have a pyramidal shape, abundant cytoplasm and are acidophil and smoothly granular in the apical and medium parts, and basophil in the basal compartment. The granulations are small, acidophilic, weakly PASpositive, disposed in the medium and apical cytoplasm. These granulations contain trypsin, amylase and lipase (6). The nuclei of the acinar pancreatic cells are small, centrally or basally localized and they present a prominent nucleolus (3). The clinical picture in case of these patients can include frequent symptoms, like: chronic abdominal pain, especially in the epigastric area, gastroesophageal reflux, nausea, vomiting, hematemesis, quirky appetite with loss of appetite, progressive weight loss,

Corresponding author:

Cristina Oana Marginean, University of Medicine and Pharmacy, 38 Gh. Marinescu Street, Targu-Mures

E-mail: marginean.oana@gmail.com 
anemia by iron deficit (4). The differential diagnosis involves other types of metaplasia, like the intestinal one. In order to confirm the diagnosis, the election method is the superior digestive endoscopy that allows in certain cases to visualize the lesion and to take multiple biopsies for the histopathological diagnosis (7). The treatment of pancreatic metaplasia is determined by the severity of the lesions. In severe cases, the surgical removal is the only option (8).

\section{CASE PRESENTATION}

We present the case of a 13-year-old patient admitted in the Gastroenterology Compartment of the Pediatrics Clinics 1 Targu-Mures, presenting recurrent abdominal pain and heartburns. The familial and personal history did not reveal relevant elements. The pathological elements pointed out by the clinical exam performed at the moment of admission were the following: influenced general status, elastic and soft abdomen, painful at palpation in the epigastric area, $45 \mathrm{~kg}$ weight.

The laboratory test did not reveal significant or specific abnormalities, but the CBC count showed lymphocytosis (41\%) with monocytosis $(8.9 \%)$, eosinophilia (4.9\%). We also identified an increased number of reticulocytes $(20 \%)$, a decreased serum level of triglycerides $(48.8 \mathrm{mg} / \mathrm{dl})$, and a value above the superior limit for calcium (Ca 2.57 $\mathrm{mmol} / \mathrm{L})$ and magnesium $(\mathrm{Mg} 1 \mathrm{mmol} / \mathrm{L})$, the rest of the parameters being in normal ranges, including the serum amylase. The paraclinical investigations, such as abdominal ultrasound did not reveal visible pathological modifications. Taking in consideration the clinical symptomatology, suggestive for a gastrointestinal disorder, we decided to perform a superior digestive endoscopy, during which we noticed the presence of gastroesophageal reflux and a granular aspect of the gastric mucosa. Therefore, we took biopsy from the antral gastric mucosa. The pathological exam performed from the biopsy revealed a fragment of pancreatic metaplasia in the gastric mucosa (Fig. 1 and 2).

Thus, we established the diagnosis of pancreatic metaplasia in the gastric mucosa and we recommended diet with the avoidance of fried food, condiments, fats, juices, and treatment with proton pump inhibitor (Nexium $40 \mathrm{mg} /$ day) and prokinetic (Motilium 2x10 mg/day) for a month. The patient's evolution was favorable.

The particularity of the case consists in the presence of pancreatic metaplasia of the gastric mucosa in a patient with recurrent abdominal pain and
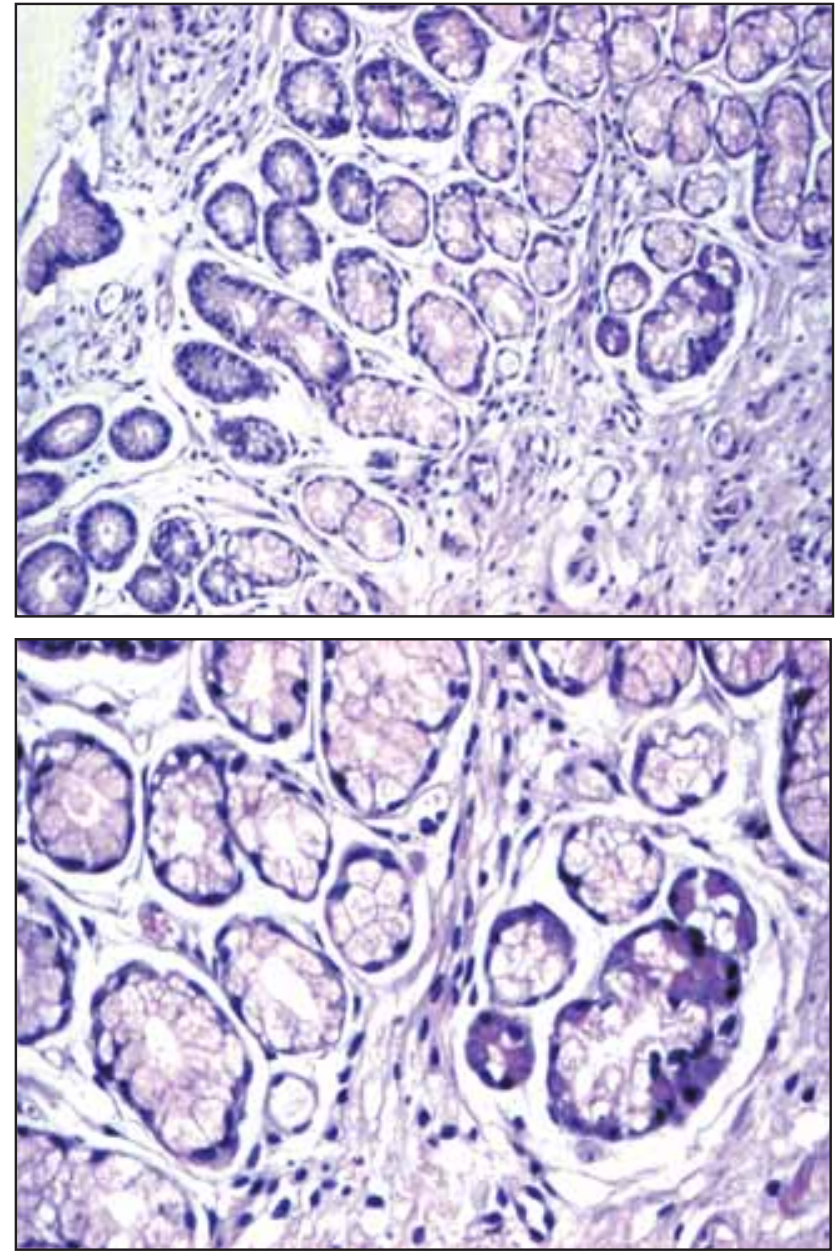

FIGURE 1 and 2. Histopathological aspect of the pancreatic metaplasia in the gastric mucosa

heartburns, without significant familial or personal history.

\section{DISCUSSIONS}

The term of pancreatic metaplasia suggests that the transforming process takes place in the postnatal life and involves a tissue which appears in a place where it shouldn't normally be. Some researchers consider that it is an adaptive process of the structures to the new conditions of the environment. In the specialty literature, more and more often is cited the fact that this transforming process develops in the structures that have been submitted to chronic trauma, infections or hormonal stimulations, therefore being submitted to continuous regeneration processes $(9,10)$. A study performed by Doglioni et al describes the fact that pancreatic metaplasia is present in approximately $12 \%$ of the cases with autoimmune gastritis that appears usually in the cardia and it coexists with other types of metaplasia, such as the intestinal one (11). Jhala NC describes in 2003, an incidence of $11 \%$ for the pancreatic metaplasia of the oxyntic mucosa, study- 
ing fragments of gastric biopsy in adult patients, among which $9 \%$ associate autoimmune gastritis, $1 \%$ of the patients present multifocal chronic atrophic gastritis and 1\% present normal gastric mucosa (5). In the case presented above, there were not identified other pathological associations of the gastric mucosa, the rest of the mucosa presenting a normal histopathological aspect. The diagnosis of pancreatic metaplasia is difficult to establish, especially when there is an abundant inflammatory process, and the acinar cells can be mistaken by the parietal ones from the inferior part of the gastric glands. In these situations, the immunohistochemical techniques are very useful, providing a considerable advantage by underlying the presence of the characteristic enzymes for the pancreatic acinar cells (3). The molecular pathogenesis of the pancreatic metaplasia is insufficiently known. Certain authors agree with the fact that pancreatic metaplasia results from an aberrant differentiation of the stem cells present in the neck of the gastric glands (8). The study performed by Integlia et al describes the clinical and pathological characteristics of 6 children aged between 8 and 18 years, with clear evidences of pancreatic metaplasia of the gastric mucosa. Among the 6 children, 2 presented gastroesophageal reflux disease and chronic abdominal pain, one presented duodenal ulcer and one associated nodular gastritis. Iron deficiency anemia was present in 4 of the 6 cases, and 3 of 4 patients with this diagnosis also presented hematemesis. All the performed biopsies were negative for Helicobacter Pylori (4). Similarly, our patient presented chronic abdominal pain and gastroesophageal reflux, and the pathological exam did not reveal the presence of Helicobacter pylori in the biopsy specimen from the gastric mucosa. Johansson $\mathrm{J}$ et al, consider that pancreatic metaplasia of the gastric mucosa can be a lesion depending on age, female gender and gastroesophageal reflux, being associated with the Helicobacter Pylori infection (12,13). Pancreatic metaplasia identified in the case presented by us developed also in female gender, being associated with gastroesophageal reflux, but without Helicobacter pylori infection. In the specialty literature, there are sources that sustain that the inlands of pancreatic acinar cells produce pancreatic enzymes, such as amylase, lipase and trypsin (3). Nevertheless, the serum amylase performed in the case of our patient was in normal ranges. The etiology of pancreatic metaplasia is still unclear, therefore further studies are needed for its clarification.

\section{CONCLUSIONS}

Pancreatic metaplasia associated with modifications of the gastric mucosa and gastroesophageal reflux were discovered endoscopically, therefore being mandatory to perform this investigation in children with recurrent abdominal pain and heartburns. The common treatment of the lesions associated to pancreatic metaplasia improved the clinical picture. The pathogenesis and the long term prognosis of the pancreatic metaplasia in the gastric mucosa are still unclear.

\section{REFERENCES}

1. Slack J.M.W. Metaplasia and somatic cell reprogramming. J Pathol 2009; 217(2):161-8.

2. Slack J.M.W, McGee J., Isaacson P., Wright N.A. Oxford Textbook of Pathology. Vol. I: Principles of Pathology, Oxford University Press 1992; 1:565-8.

3. Doglioni C., Laurino L., Del Tos A. et al. Pancreatic (acinar) metaplasia of the gastric mucosa. Histology, ultrastrucuture, immunocytochemistry, and clinicopathologic correlations of 101 cases. Am J Surg Pathol 1993; 17:1134-43.

4. Integlia M.J., Krishnamurthy S., Berhane R., Grand R.J., Dayal Y. Pancreatic Metaplasia of the Gastric Mucosa in Pediatric Patients. Am J Gastroenterol 1997; 92(9):1553-6.

5. Jhala N.C., Montemor M., Jhala D. et al. Pancreatic acinar cell metaplasia in autoimmune gastritis. Arch Pathol Lab Med 2003; 127(7):854-7.

6. Buettner M., Dimmler A., Magener A. et al. Gastric PDX-1 expression in pancreatic metaplasia and endocrine cell hyperplasia in atrophic corpus gastritis. Mod Pathol 2004; 17(1):56-61.

7. Edlund H. Pancreatic organogenesis - developmental mechanisms and implications for therapy. Nat Rev Genet 2002; 3(7):524-32.

8. Krishnamurthy S., linteglia M.J., Grand R.J., Dayal Y. Pancreatic acinar cell clusters in pediatric gastric mucosa. Am J Surg Pathol 1998; 22(1):100-5.

9. Levin J.K., Riddel R.H., Weinstein W.M. Gastrointestinal Pathology and Its Clinical Implications. New York, Igaku-Shoin, 1992:616.

10. Jhala N., Montemor J., Jhala D. et al. Pancreatic acinar cells in gastricoxyntic mucosa. Mod Pathol 1990; 12:76A.

11. El-Zimaity H.M., Verghese V.J., Ramchatesingh J., Graham D.Y. The gastric cardia in gastro-oesophageal disease. J Clin Pathol 2000; 53(8):619-25.

12. Johansson J., Hakansson H.O., Mellblom L. et al. Pancreatic acinar metaplasia in the distal oesophagus and the gastric cardia: prevalence, predictors and relation to GORD. J Gastroenterol 2010; 45(3):291-9.

13. Edlund H. Pancreatic organogenesis - developmental mechanisms and implications for therapy. Nat Rev Genet, 2002; 3(7):524-32. 